\title{
The Risk Factors of the Progression of
}

\section{Rhegmatogenous Retinal Detachment on Patients with the Fourteen-day Quarantine in the Early Period of COVID-19 Outbreak}

\section{Meng Zhao}

Beijing Tongren Eye Center, Beijing Key Laboratory of Ophthalmology and Visual Science, Beijing Tongren Hospital, Capital Medical University.

Jipeng Li

Beijing Tongren Eye Center, Beijing Key Laboratory of Ophthalmology and Visual Science, Beijing Tongren Hospital, Capital Medical University.

\section{Haicheng She}

Beijing Tongren Eye Center, Beijing Key Laboratory of Ophthalmology and Visual Science, Beijing Tongren Hospital, Capital Medical University.

Ningpu Liu ( $\square$ nliu001@gmail.com )

Beijing Tongren Eye Center, Beijing Key Laboratory of Ophthalmology and Visual Science, Beijing Tongren Hospital, Capital Medical University.

\section{Research Article}

Keywords: rhegmatogenous retinal detachment, COVID-19, postponed surgery

Posted Date: February 4th, 2021

DOl: https://doi.org/10.21203/rs.3.rs-149017/v1

License: (a) This work is licensed under a Creative Commons Attribution 4.0 International License. Read Full License 


\section{Abstract}

\section{Backgrounds: The COVID-19 pandemic has great impact on hospitals and patients. The fourteen-day quarantine caused surgery of RRD postponed. To explore the risk factors of rhegmatogenous retinal}

detachment (RRD) progression in a group of patients whose surgery was postponed during the top-level emergency response of COVID-19.

Methods: Retrospective case series study. The information on RRD patients who received surgeries in Beijing Tongren Hospital's retina service from February 16, 2020, to April 30, 2020 has been collected retrospectively. The history, manifestation on presentation and admission, and progression of RRD were recorded. RRD progression was defined as the presence of either choroidal detachment (CD) or proliferative vitreoretinopathy (PVR) progression during the quarantine period. Risk factors were analyzed using the Cox proportional hazards model, survival analysis, and logistic regression.

Results: There were 79 cases enrolled in this study. The median time from the patients' presentation at the clinic to admission for surgery was 14 days (3-61 days). There were 70 cases ( $88.6 \%)$ who did not present to the hospital within one week of the onset of visual symptoms. There were 69 (87.3\%) macularoff cases at the presentation. There were 27 (34.2\%) cases combined with choroidal detachment. There were 49 (62.0\%) cases with PVR B, 22 (27.8\%) cases with PVR C, 4 (5.1\%) cases with PVR D, and 4 (5.1\%) cases with PVR A. After the 14-day quarantine, 21 (26.6\%) cases showed RRD progression, and 9 cases showed RD regression at the time of surgery. Neither the time of onset of the visual symptom $(p=0.46)$ nor the time between presentation and admission $(p=0.31)$ was significantly different between the patients with RRD progression and patients without RRD progression. The combination of CD $(3.61,1.86-$ $6.99, p=0.001)$ and retinal breaks located posterior to the equator $(3.78,1.25-11.45, p=0.02)$ were factors related to the progression of RRD.

Conclusions: In the cases enrolled in our study during the COVID-19 outbreak period, the RRD progression risk factors included a combination of $C D$ and retinal breaks posterior to the equator. Ophthalmologists should schedule the surgeries for RRD patients with these signs as soon as possible.

\section{Background}

Rhegmatogenous retinal detachment (RRD) is a sight-threatening ocular disease requiring surgical intervention ${ }^{[1]}$; it has a prevalence of $6.3-17.9 / 100$ thousand people ${ }^{[2]}$. It can lead to the development of choroidal detachment (CD) $[3,4]$, the progression of proliferative vitreoretinopathy (PVR) $[5,6]$, and irreversible visual damage ${ }^{[7]}$ if it is not treated in time. It has been reported that the poor visual acuity outcome is related to the delay of surgery in RRD patients ${ }^{[8,9]}$, and it is recommended to perform surgery within three days of the onset of symptoms to achieve a better visual outcome[8, 10-12]. The outcome of long-standing RRD is not well addressed.

The outbreak of the highly contagious coronavirus disease (COVID-19) has a significant impact on health services worldwide. In China, Beijing upgraded its emergency response to top-level three days after ten 
COVID-19 cases were first confirmed in February 2020. A 14-day self-quarantine for all patients who were going to be admitted was announced later. In response to the pandemic, most elective surgeries were postponed except for urgent surgeries $[1,13,14]$. RRD surgeries were carried out in patients who had a negative screening result for COVID-19. Since there was no laboratory test of the COVID-19 virus in the early days of the pandemic, the COVID-19 screening in our center included a 14-day self-quarantine with daily self-reported normal body temperature, as well as a normal blood cell count and pulmonary CT scan before admission. During the period, the RRD patients could only visit the emergency room for diagnosis and had to wait at least14 days to be operated on in the ward.

We have tried to investigate the progression of RRD after a 14-day quarantine. We looked for the risk factors related to the progression of RRD.

\section{Methods}

We retrospectively reviewed the RRD cases who underwent surgery from February 16, 2020, to April 30, 2020, in Beijing Tongren Eye center. This study was approved by the Ethics Committee of Beijing Tongren Hospital and adhered to the tenets of the Declaration of Helsinki.

All patients were registered as soon as they were diagnosed at the outpatient clinics. They were asked to report their daily temperature and quarantine in Beijing. After the quarantine, patients were informed to go to the hospital to screen for the COVID-19, take laboratory tests, and receive ocular examinations for surgery. We collected the preoperative characteristics of the patients. These characteristics included age, gender, presurgery waiting times (from visual symptom to surgery), quarantine time (from being diagnosed to surgery), history of previous eye trauma, previous surgical history, preoperative visual acuity (VA), lens status, the extent of retinal detachment, location of retina breaks, proliferative vitreoretinopathy (PVR), choroidal detachment (CD) and congenital vitreoretinal diseases (coloboma, familial exudative vitreoretinopathy et al.). The changes of RD at admission compared to the record of the first time at the outpatient clinic were recorded. The focus was placed on the development and progression of CD and PVR. PVR progression was defined as the growth of membranes on both surfaces of the detached retina and on the posterior surface of the detached vitreous gel ${ }^{[5]}$. CD was defined as presenting with low intraocular pressure, anterior chamber inflammation, choroidal detachment were found by either indirect ophthalmoscopy or B scan[4]. RD progression was recorded as at least one of the following conditions developed at the time of admission compared to the initial presentation: 1) development of $C D ; 2$ ) progression of PVR; 3 ) the extent of RRD progressed to $3 / 4$ quadrants of 4 quadrants RRD.

Statistical analysis was performed using R version 3.20 (http://www. R-project.org). Patient characteristics were retrieved from their medical charts and recorded in Epidata Entry Clientversion2.0.3.15 (http://epidata.dk). Mean and standard deviation (SD) were calculated for continuous variables with a normal distribution. Median with quartiles was calculated for continuous variables with a non-normal distribution. T-test or Mann-Whitney $U$ test was carried out for continuous variables. Chi-square test or Fisher's exact test was carried out for discrete data. The Cox proportional- 
hazards model was used to investigate the association between patients' quarantine time and several characteristics that may be related to RD progression. The survival analysis, Kaplan-Meier curve, and logrank test were performed on the related factors. The binary backward stepwise logistic regression model was carried out to explore the potential risk factors at the initial presentation. One variable was included or excluded from the model each time by comparing the Akaike information criterion (AIC) value, and the model with the lowest AIC was chosen.

\section{Results}

Seventy-nine patients were enrolled in this study, with a majority of male patients (70.9\%). The average age was $49.8 \pm 15.7(12-74)$ years old. The median quarantine time was 14 days (3-61, IQR 12). The median time between the onset of symptoms and operation was 28 days (7- 336, IQR 35). There were 70 $(88.6 \%)$ patients who failed to present to the hospital within one week after the onset of their visual symptoms.

\section{Basic characteristics at presentation}

There were 45 (57.0\%) patients with primary RRD, 27 (34.2\%) patients with RRD-CD, five patients (6.3\%) with recurrent RRD (one previously had scleral buckling, and four had pars plana vitrectomy (PPV) and silicone oil tamponade), and two patients (2.5\%) with combined coloboma.

The percentage of pseudophakic eyes and PM was 18 (22.8\%), 36.7\%(29/79), respectively. Six patients had a history of PPV, while five patients had a history of scleral buckling. There were 44 (55.7\%) patients whose preoperative VA was less than $0.02,28(35.5 \%)$ patients whose preoperative VA was between 0.02 and 0.4 , and seven (8.9\%) patients whose preoperative VA was equal to or greater than 0.5 .

There were $42(53.2 \%)$ patients with four quadrants RD. There were $68 \otimes 86.1 \%$ \patients with a macular-off RD.

There were 49 (62.0\%) patients with PVR B, 22 (27.8\%) patients with PVR C, four (5.1\%) patients with PVR D, and four (5.1\%) patients with APVR. The prevalence of PVR C-D and APVR was higher in patients with RRD-CD than patients with $\operatorname{RRD}(44.1 \%, 31.1 \%, p=0.01)$.

Thirty-seven (46.8\%) patients' retinal breaks were located anterior to the equator, 38 (48.1\%) patients' retinal breaks were located posterior to the equator, and four (5.1\%) patients had a macular hole (one of them combined with tear which was posterior to the equator). RRD-CD prevalence was higher in patients with retinal breaks located posterior to the equator than patients with retinal breaks located anterior to the equator $(55.9 \%, 22.2 \%, p=0.01)$.

\section{Changes of RRD at admission}


Twenty-one (26.6\%) patients had a progression of RD after quarantine. Among them, 16 RRD-CD patients had a progression of PVR (4 patients from $C 1$ to $D 2,6$ patients from $C 1$ to $C 3$, and 2 recurrent patients with anterior PVR), and 5 RRD patients developed CD (1 patient had a simultaneous progression of PVR). Nine $(11.4 \%)$ patients had a regression of RD, while 49 (70.6\%) patients had no significant RD progression, including 11 RRD-CD patients.

Fifty-seven patients received PPV, while 22 patients received scleral buckling. Among the patients who received PPV, ten patients had preoperative posterior vitreous detachment, 25 patients whose PVD was easily induced during vitrectomy, and 28 patients had sticky vitreous, which was hard to peal during vitrectomy.

\section{Factors that may be related to the progression of RD (Table 1)}

We divided the patients into two groups based on whether RD progressed at admission. There was a significant difference between the two groups in terms of the following factors:: gender $(p=0.04)$, the combination of $\operatorname{CD}(p<0.001)$, previous history of vitrectomy $(p=0.04)$, location of retinal breaks $(p<$ $0.001)$, macular hole $(p=0.03)$, macular detachment $(p=0.05)$, sticky vitreous during vitrectomy $(p<$ $0.001)$, and VA distribution $(p=0.04)$. There was not a significant difference in quarantine time $(p=0.46)$ or the time between the onset of symptoms and presentation $(p=0.31)$ in the two groups (Table 1$)$. 
Table 1

Initial characteristics of RRD patients enrolled

\begin{tabular}{|c|c|c|c|}
\hline & $\begin{array}{l}\text { Patients with RD } \\
\text { progression (21) }\end{array}$ & $\begin{array}{l}\text { Patients without RD } \\
\text { progression(58) }\end{array}$ & $\begin{array}{l}p \\
\text { value }\end{array}$ \\
\hline Age $($ mean $\pm S B)$ & $51.1 \pm 12.2$ & $49.3 \pm 16.9$ & 0.62 \\
\hline Gender (male, n, \%) & $19,90.5 \%$ & $37,63.8 \%$ & 0.04 \\
\hline $\begin{array}{l}\text { Time between diagnosis and surgery } \\
\text { (median, range,day) }\end{array}$ & $18(3-61)$ & $13(11-75)$ & 0.46 \\
\hline $\begin{array}{l}\text { Time between onset of symptom and } \\
\text { surgery (median, range,day) }\end{array}$ & $28(7-84)$ & $28(7-336)$ & 0.31 \\
\hline Diagnosis & & & \multirow{5}{*}{$<.001 *$} \\
\hline $\operatorname{RRD}(\mathrm{n}, \%) \square$ & $2,9.5 \%$ & $42,72.4 \%$ & \\
\hline RRD-CD $(\mathrm{n}, \%)$ & $14,66.7 \%$ & $14,24.1 \%$ & \\
\hline Recurrent RRD(n,\%) & $3,14.2 \%$ & $2,1.7 \%$ & \\
\hline Combined coloboma(n,\%) & $2,9.5 \%$ & 0 & \\
\hline pseudophakic(n,\%) & $7,33.3 \%$ & $11,19.0 \%$ & $0.22^{\star}$ \\
\hline PM $(n, \%)$ & $8,38.1 \%$ & $21,36.2 \%$ & $1 *$ \\
\hline Previous PPV(n,\%) & $4,19.0 \%$ & $2,3.4 \%$ & 0.04 * \\
\hline Previous SB(n,\%) & $1,4.8 \%$ & $4,6.9 \%$ & 1 * \\
\hline Location of retinal break & & & 0.03 \\
\hline Anterior to equator $(n, \%)$ & $1,4.8 \%$ & $36,62.1 \%$ & \multirow{2}{*}{$\begin{array}{l}<.001 \\
0.00\end{array}$} \\
\hline Posterior to equator ( $\mathrm{n}, \%)$ & $17,81.0 \%$ & $21,36.2 \%$ & \\
\hline Macular hole(n,\%) & $3,14.3 \%$ & $1,1.7 \%$ & 0.05 \\
\hline PVR & & & \multirow[t]{5}{*}{0.35} \\
\hline$B(n, \%)$ & $12,57.1 \%$ & $37,63.8 \%$ & \\
\hline$C(n, \%)$ & $5,23.8 \%$ & $17,29.3 \%$ & \\
\hline$D(n, \%)$ & $2,9.5 \%$ & $2,3.4 \%$ & \\
\hline $\operatorname{APVR}(\mathrm{n}, \%)$ & $2,9.5 \%$ & $2,3.4 \%$ & \\
\hline Macular-off(n,\%) & $21,100 \%$ & $48,82.8 \%$ & 0.05 \\
\hline PVD & & & $<.001 *$ \\
\hline Presurgery VA & & & 0.04 \\
\hline
\end{tabular}




\begin{tabular}{|llll|}
\hline & $\begin{array}{l}\text { Patients with RD } \\
\text { progression (21) }\end{array}$ & $\begin{array}{l}\text { Patients without RD } \\
\text { progression(58) }\end{array}$ & $\begin{array}{l}\boldsymbol{p} \\
\text { value }\end{array}$ \\
\hline Less than $0.02(n, \%)$ & $14,66.7 \%$ & $21,36.2 \%$ & \\
\hline$[0.02-0.1)(n, \%)$ & $7,33.3 \%$ & $30,51.7$ \\
\hline$>=0.5(n, \%)$ & 0 & $7,12.1 \%$ \\
\hline
\end{tabular}

The Cox proportional-hazards model showed that, patients with RRD-CD at presentation were 3.61 times more likely to have RD progression $(1.86-6.99, p=0.001)$ than patients without CD; patients with retinal breaks located posterior to the equator were 3.78 times more likely to have RD progression (1.25-11.45, $p=0.02$ ) compared to patients with retinal breaks located anterior to the equator (Wald test $\mathrm{F}=27.64, p<$ 0.001 , LogRank test $F=46.47, p<0.001$ ). The median survival time for RD progression was 61 days after the onset of symptoms (Fig. 1-2). The log-rank test in survival analysis showed that the median survival time for RD progression was 13.5 days in patients with macular hole, 22 days in patients with retinal breaks located posterior to the equator, 14 days in patients with giant tears, 18.5 days in patients with unattached retina after surgery, 22 days in RRD-CD patients, and 18 days in patients with sticky vitreous.

The logistic regression analysis showed that female $(25,2.04-1000, p=0.03)$, combination with CD $(5.22,2.18-17.66, p=0.001)$, and retinal breaks located posterior to the equator $(17.91,3.44-224.56, p=$ 0.004 ) were factors that may be related to RD progression ( $A I C=52.23, A U C=0.918$ ).

\section{Discussion}

The 14-day quarantine during the early period of the COVID-19 pandemic provided us the chance to observe the short natural course of RRD when the surgery had to be delayed. We found that $26.6 \%$ of patients had RD progression, while $11.4 \%$ of patients had RD regression, $70.6 \%$ of patients had no significant RD progression after the quarantine. We further investigated the initial characteristics that may be related to the RD progression.

Previous reports focusing on RD progression find that prolonged presurgery waiting time is related to the development of macular-off RD[12], irreversible macular damage[15-17], PVR progression[18], and development of $\mathrm{CD}[19]$. We chose PVR progression, the progression of RD's extent, and CD's development as the signs for RD progression. Since the prevalence of macular detachment (86.1\%) in our group of patients was much higher than what was reported in previous studies ${ }^{[10,20]}$, which focused on RD's progression from macular-on to macular-off, we could not use the development of macular-off as a sign for RD progression.

$C D$ is related to retinal detachment surgery failure[21], and its prevalence is $8.6 \%[22]-18.79[23] \%$ in Chinese RRD patients. We had a much higher prevalence of RRD-CD in our group of patients at $34.2 \%$. The prevalence of PVR C-D is higher in RRD-CD patients at 28[24]-66.9\%[25] compared to RRD patients in previous reports. PVR progression was found in 16 RRD-CD patients. We found a similar result of PVR C$\mathrm{D}$ at $44.1 \%$ in RRD-CD patients and $33.1 \%$ in RRD patients. We found a retinal break located posterior to 
the equator was related to the RRD-CD development, similar to previously reported[22]. Our result showed RRD-CD patients were much more likely to experience RD progression shortly after diagnosis. The median time for RD progression in RRD-CD patients was 22 days after the onset of the symptom. Our result suggested that patients with RRD-CD should be operated on without delay in case of fast progression of RD.

PVR is the most common cause of RRD surgery failure $[5,21]$. The progression of PVR is reported to be related to the following conditions: a giant tear, a long course of RRD, vitreous hemorrhage, pseudophakic eye, the combination of $C D$, gas tamponade, and cryotherapy $[6,26]$. The chance of developing PVR B-C has been reported to be higher in patients whose presurgery waiting times are longer than 40 days[18]. The prevalence of PVR C-D and APVR in our study was $38.0 \%$. It was similar to what was previously reported at $12.9-21.6 \%[27]$ in patients with scleral buckling and $26.9-41.6 \%[18,27,28]$ in patients with PPV. The initial presentation of PVR in our group of patients was not related to RD progression. The reasons may be related to the fact that the presurgery waiting time was too short to observe PVR progression. In other words, except for patients with $C D$, the presence of PVR was not related to short-time $\mathrm{RD}$ progression.

In addition to the presence of $C D$ and the location of a retinal break, the survival analysis found that the median survival time for RD progression was short in the following conditions in survival analysis: macular hole (13.5 days), giant tear (14 days), combining with coloboma (6 days), and recurrent RD (18.5 days). We failed to show the relationship between the factors mentioned above to RD progression due to the small sample size. We still need to pay extra attention to RRD patients with the conditions mentioned above in the case of RD progression.

The limitation of the study was due to the retrospective character and the limited case number. During the COVID-19 pandemic, we gained the chance to observe the natural course of RRD. The prolonged presurgery waiting time may lead to a high prevalence of CD, PVR C-D, and macular-off. Since there was a high prevalence of 4 quadrants RD in our group of patients, the presence of more challenging cases at the initial presentation makes it impossible to progress to a more severe condition in a short follow-up time. More cases with newly developed RRD with short presurgery waiting time should be involved to see the risk factors for RD progression. Also, we did not report the outcomes of surgeries. We can not show the impact of prolonged presurgery waiting time on the prognosis of RRD.

\section{Conclusion}

We have reported a group of RRD patients with a high prevalence of PVR C-D, CD, and macular-off who underwent surgery during the COVID-19 pandemic. After the quarantine, some of the patients had RD progression. Ophthalmologists should pay more attention to RRD patients with CD or retinal breaks located posterior to the equator in case of RD progression shortly after the diagnosis.

\section{List Of Abbreviations}


Akaike information criterion AIC

choroidal detachment CD

coronavirus disease COVID-19

visual acuity BCVA

intraocular pressure $\quad \mathrm{IOP}$

intraocular lens $\mathrm{IOL}$

macular hole $\mathrm{MH}$

pathological myopia PM

posterior vitreous detachment

PVD

proliferative vitreoretinopathy PVR

receiver operating characteristic curve ROC curve

rhegmatogenous retinal detachment RRD

retinal detachment $\mathrm{RD}$

standard deviation SD

\section{Declarations}

\section{Ethics declarations}

\section{Ethics approval and consent to participate:}

This study was conducted in accordance with the Declaration of Helsinki and approved by the medical ethics committee of Beijing Tongren Hospital. The reference number is TRECKY 2020-071. The need for written informed consent was waived because of the retrospective design and use of deidentified patients data. This was also approved by the medical ethics committee of Beijing Tongren Hospital

\section{Consent for publication:}

Not applicable. 


\section{Availability of data and material}

The datasets used and/or analysed during the current study are available from the corresponding author on reasonable request.

\section{Competing interests}

The authors declare that they have no competing interests.

\section{Funding}

Not applicable.

\section{Author information}

\section{Affiliations}

Beijing Tongren Eye Center, Beijing Key Laboratory of Ophthalmology and Visual Science, Beijing Tongren Hospital, Capital Medical University. No1. Dongjiaominxiang street, Dongcheng District, Beijing,100730, China

Meng Zhao, Jipeng Li, Haicheng She, Ningpu Liu

\section{Authors' contributions}

All authors read and approved the final manuscript. M Zhao collected and analyzed the data, she was the one major contributor in writing the manuscript. HC She interpreted the data she was one major contributor in writing and reviewing the manuscript. JP Li collected the data and reviewed the manuscript. NPL reviewed the manuscript and contribute in writing the manuscript.

\section{Acknowledgments}

We acknowledge all the healthcare workers in our department who made the RRD service possible during the pandemic. Dr. Ningpu Liu is the leader of the RRD service during the pandemic. During the Covid-19 pandemic, his decisions and actions were full of intelligence and courage, caring for both the patients and colleagues, showing what a real doctor should do. It is a great honor to work with him and work in this team to provide service for patients. 


\section{References}

1. (ASRS) ASoRS: American Society of Retina Specialists (ASRS) Member Alert Regarding the COVID19 Pandemic. 2020.

2. Mitry D, Charteris DG, Fleck BW, Campbell H, Singh J: The epidemiology of rhegmatogenous retinal detachment: geographical variation and clinical associations. Br J Ophthalmol 2010; 94(6):678684.https://doi.org/ 10.1136/bjo.2009.157727.

3. Gottlieb F: Combined choroidal and retinal detachment. Arch Ophthalmol 1972; 88(5):481486.https://doi.org/ 10.1001/archopht.1972.01000030483003.

4. Seelenfreund MH, Kraushar MF, Schepens CL, Freilich DB: Choroidal detachment associated with primary retinal detachment. Arch Ophthalmol 1974; 91(4):254-258.https://doi.org/ 10.1001/archopht.1974.03900060264003.

5. Pastor JC: Proliferative vitreoretinopathy: an overview. Surv Ophthalmol 1998; 43(1):318.https://doi.org/ 10.1016/s0039-6257(98)00023-x.

6. Nagasaki H, Shinagawa K, Mochizuki M: Risk factors for proliferative vitreoretinopathy. Prog Retin Eye Res 1998; 17(1):77-98.https://doi.org/ 10.1016/s1350-9462(97)00007-4.

7. Ivanisević M: The natural history of untreated rhegmatogenous retinal detachment. Ophthalmologica 1997; 211(2):90-92.https://doi.org/ 10.1159/000310766.

8. Frings A, Markau N, Katz T, Stemplewitz B, Skevas C, Druchkiv V, Wagenfeld L: Visual recovery after retinal detachment with macula-off: is surgery within the first $72 \mathrm{~h}$ better than after? $\mathrm{Br} \mathrm{J}$ Ophthalmol 2016; 100(11):1466-1469.https://doi.org/ 10.1136/bjophthalmol-2015-308153.

9. Kim JD, Pham HH, Lai MM, Josephson JW, Minarcik JR, Von Fricken M: Effect of symptom duration on outcomes following vitrectomy repair of primary macula-off retinal detachments. Retina 2013; 33(9):1931-1937.https://doi.org/ 10.1097/IAE.0b013e3182877a27.

10. Hajari JN, Kyhnel A, Bech-Azeddine J, la Cour M, Kiilgaard JF: Progression of foveola-on rhegmatogenous retinal detachment. Br J Ophthalmol 2014; 98(11):1534-1538.https://doi.org/ 10.1136/bjophthalmol-2014-305157.

11. Wykoff CC, Smiddy WE, Mathen T, Schwartz SG, Flynn HW, Jr., Shi W: Fovea-sparing retinal detachments: time to surgery and visual outcomes. Am J Ophthalmol 2010; 150(2):205210.e202.https://doi.org/ 10.1016/j.ajo.2010.03.002.

12. van Bussel EM, van der Valk R, Bijlsma WR, La Heij EC: Impact of duration of macula-off retinal detachment on visual outcome: a systematic review and meta-analysis of literature. Retina 2014; 34(10):1917-1925.https://doi.org/ 10.1097/iae.0000000000000296.

13. Ophthalmology AAo: Recommendations for urgent and nonurgent patient care. 2020.

14. Surgeons ACo: COVID-19: Guidance for Triage of Non-Emergent Surgical Procedures. 2020.

15. Park DH, Choi KS, Sun HJ, Lee SJ: FACTORS ASSOCIATED WITH VISUAL OUTCOME AFTER MACULA-OFF RHEGMATOGENOUS RETINAL DETACHMENT SURGERY. Retina 2018; 38(1):137147.https://doi.org/ 10.1097/iae.0000000000001512. 
16. Mitry D, Awan MA, Borooah S, Syrogiannis A, Lim-Fat C, Campbell H, Wright AF, Fleck BW, Charteris DG, Yorston $D$ et al: Long-term visual acuity and the duration of macular detachment: findings from a prospective population-based study. Br J Ophthalmol 2013; 97(2):149-152.https://doi.org/ 10.1136/bjophthalmol-2012-302330.

17. Enders P, Schick T, Kemper C, Schaub F, Fauser S: Effect of Symptom Duration until Surgery on FirstYear Outcome in Pseudophakic Primary Rhegmatogenous Retinal Detachment. Ophthalmologica 2017; 237(2):73-77.https://doi.org/ 10.1159/000455805.

18. Tseng W, Cortez RT, Ramirez G, Stinnett S, Jaffe GJ: Prevalence and risk factors for proliferative vitreoretinopathy in eyes with rhegmatogenous retinal detachment but no previous vitreoretinal surgery. Am J Ophthalmol 2004; 137(6):1105-1115.https://doi.org/ 10.1016/j.ajo.2004.02.008.

19. Gu YH, Ke GJ, Wang L, Gu QH, Zhou EL, Pan HB, Wang SY: Risk factors of rhegmatogenous retinal detachment associated with choroidal detachment in Chinese patients. Int J Ophthalmol 2016; 9(7):989-993.https://doi.org/ 10.18240/ijo.2016.07.09.

20. Kontos A, Williamson TH: Rate and risk factors for the conversion of fovea-on to fovea-off rhegmatogenous retinal detachment while awaiting surgery. Br J Ophthalmol 2017; 101(8):10111015.https://doi.org/ 10.1136/bjophthalmol-2016-309178.

21. Adelman RA, Parnes AJ, Michalewska Z, Ducournau D: Clinical variables associated with failure of retinal detachment repair: the European vitreo-retinal society retinal detachment study report number 4. Ophthalmology 2014; 121(9):1715-1719.https://doi.org/ 10.1016/j.ophtha.2014.03.012.

22. Yu Y, An M, Mo B, Yang Z, Liu W: Risk factors for choroidal detachment following rhegmatogenous retinal detachment in a chinese population. BMC Ophthalmol 2016; 16:140.https://doi.org/ 10.1186/s12886-016-0319-9.

23. Li Z, Li Y, Huang X, Cai XY, Chen X, Li S, Huang Y, Lu L: Quantitative analysis of rhegmatogenous retinal detachment associated with choroidal detachment in Chinese using UBM. Retina 2012; 32(10):2020-2025.https://doi.org/ 10.1097/IAE.0b013e3182561f7c.

24. Denwattana A, Prakhunhungsit S, Thoongsuwan S, Rodanant N, Phasukkijwatana N: Surgical outcomes of preoperative steroid for rhegmatogenous retinal detachment with associated choroidal detachment. Eye (Lond) 2018; 32(3):602-607.https://doi.org/ 10.1038/eye.2017.262.

25. Yu Y, Yue Y, Tong N, Zheng P, Liu W, An M: Anatomic Outcomes and Prognostic Factors of Vitrectomy in Patients with Primary Rhegmatogenous Retinal Detachment Associated with Choroidal Detachment. Curr Eye Res 2019; 44(3):329-333.https://doi.org/ 10.1080/02713683.2018.1540705.

26. Girard P, Mimoun G, Karpouzas I, Montefiore G: Clinical risk factors for proliferative vitreoretinopathy after retinal detachment surgery. Retina 1994; 14(5):417-424.https://doi.org/ 10.1097/00006982$199414050-00005$.

27. Heimann H, Bartz-Schmidt KU, Bornfeld N, Weiss C, Hilgers RD, Foerster MH: Scleral buckling versus primary vitrectomy in rhegmatogenous retinal detachment: a prospective randomized multicenter clinical study. Ophthalmology 2007; 114(12):2142-2154.https://doi.org/ 10.1016/j.ophtha.2007.09.013. 
28. Tosi GM, Balestrazzi A, Baiocchi S, Tarantello A, Cevenini G, Marigliani D, Simi F: COMPLEX RETINAL DETACHMENT IN PHAKIC PATIENTS: Previtrectomy Phacoemulsification Versus Combined Phacovitrectomy. Retina 2017; 37(4):630-636.https://doi.org/ 10.1097/iae.0000000000001221.

\section{Figures}

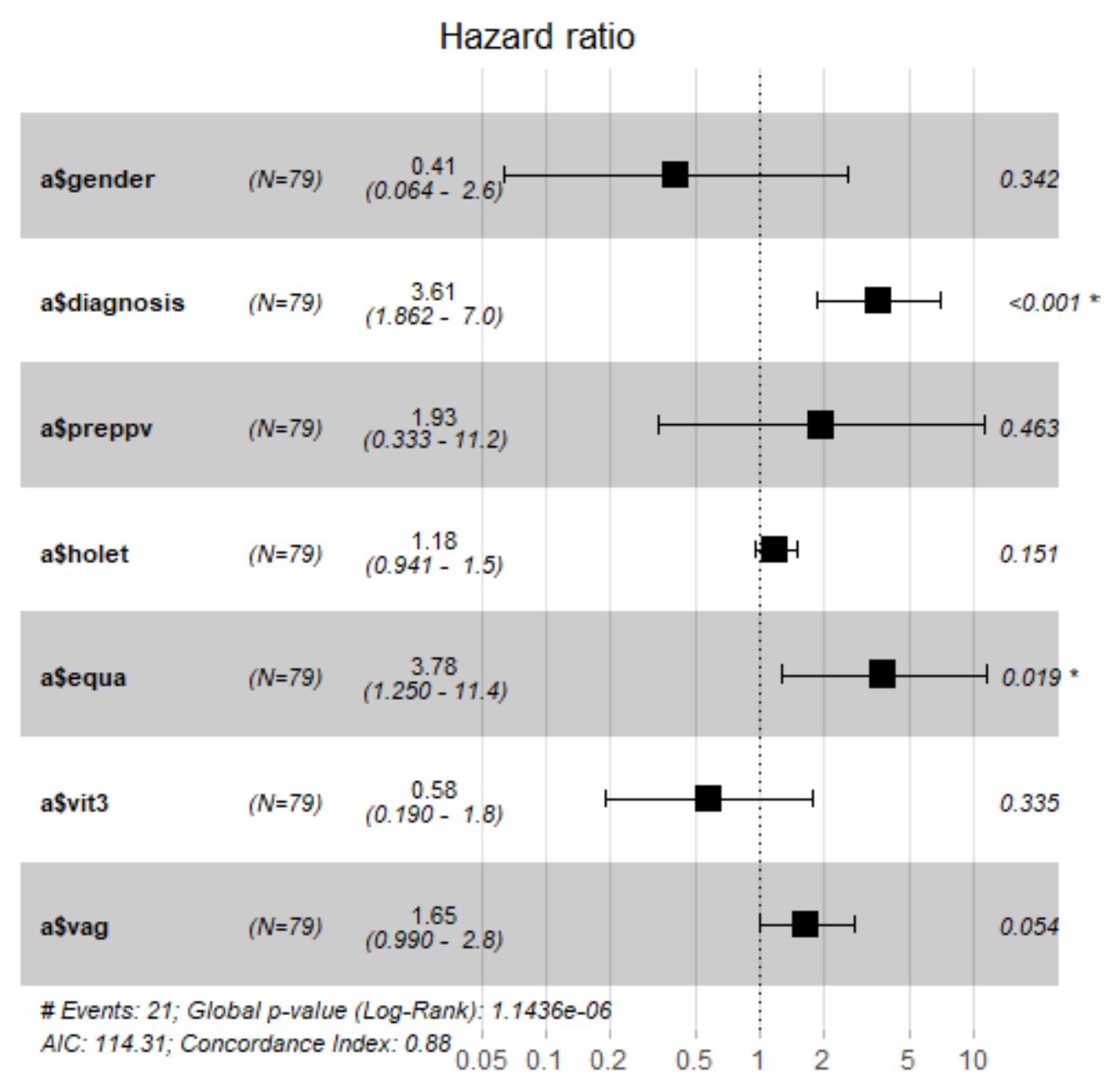

\section{Figure 1}

The tree-plot of Cox proportional-hazards model for RD progression The patients with RRD-CD at presentation were 3.61 times more likely to have RD progression $(1.86-6.99, p=0.001)$ than patients without $C D$; patients with retinal breaks located posterior to the equator were 3.78 times more likely to have RD progression $(1.25-11.45, p=0.02)$ compared to patients with retinal breaks located anterior to the equator (Wald test $F=27.64, p<0.001$, LogRank test $F=46.47, p<0.001$ ) 


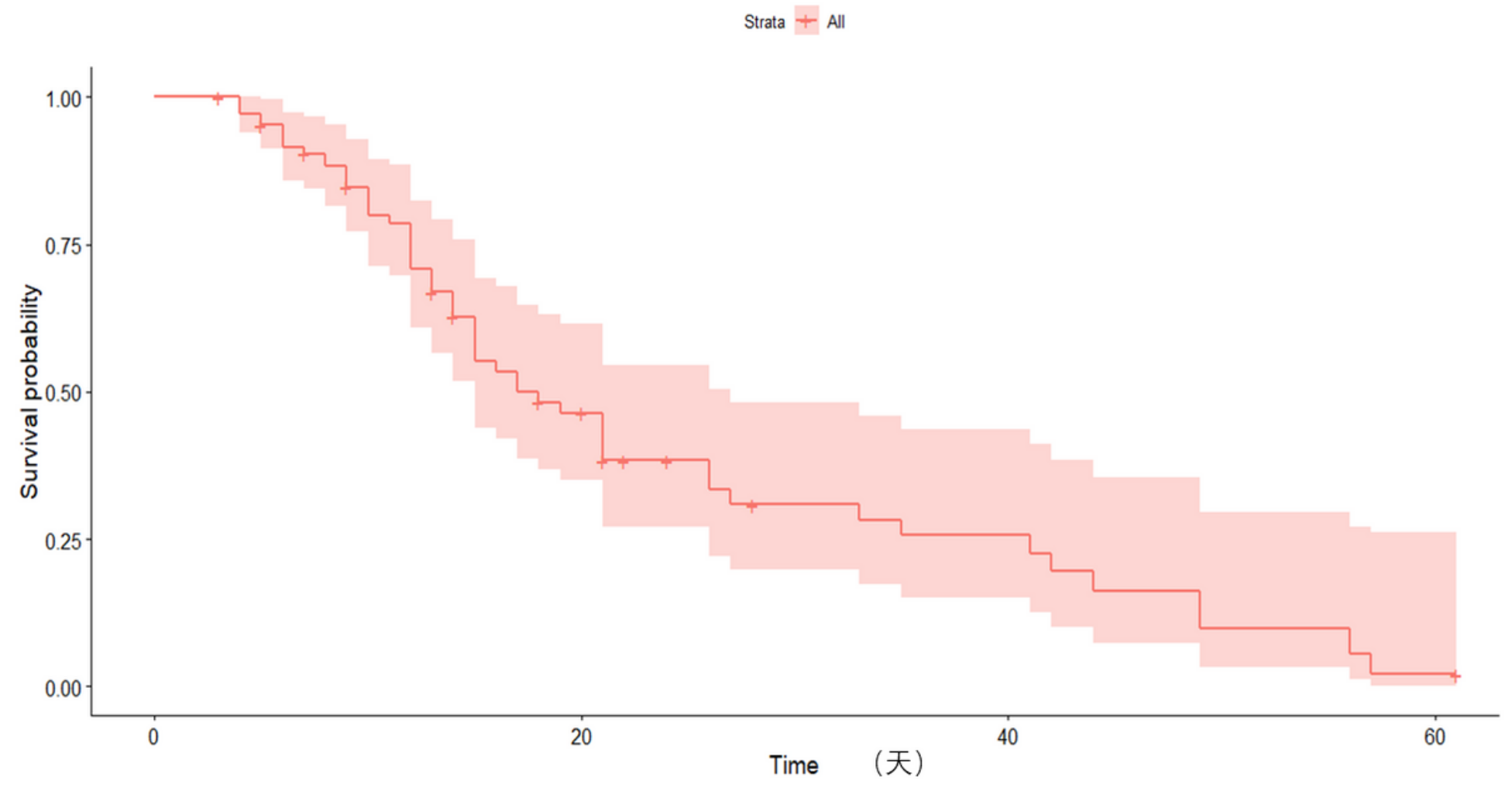

Figure 2

Kaplan-Meier survival analysis curve for the RD progression The median survival time for RD progression was 61 days after the onset of symptoms. 